\title{
An unusual cause of delayed full enteral feeding and prolonged hospital stay in a newborn with gastroschisis: Congenital Hypothyroidism
}

\author{
Sevgi Buyukbese Sarsu ${ }^{1 *}$
}

\begin{abstract}
Gastroschisis, is a prevalently encountered congenital disease of the newborns where intraabdominal organs protrude through a full-thickness defect in the anterior abdominal wall without an overlying sac. In recent years, an increase in the global incidence of gastroschisis has been reported. Although its etiology is not known fully, it is usually associated with young maternal age. Delay in the transition to full-calorie enteral feeding (ENT) in a newborn with gastroschisis is a result of bowel dysfunction which is a major morbidity developing due to exposure of bowels to amniotic fluid. Consequently, newborn may require long-term TPN treatment, and sepsis may onset secondary to prolonged mechanical ventilation, and TPN treatment, and infection,.

Global incidence of congenital hypothyroidism $(\mathrm{CH})$ in live births is 1:3000-4000. Widely encountered symptoms may include prolonged jaundice, constipation, distended abdomen, and poor feeding. The diagnosis should be confirmed by finding an elevated serum TSH and low T4 or free T4 level. In the literature, transient hypothyroidism has been reported in cases who underwent only silo operation, and povidone iodine dressings,. Association of hypothyroidism with delayed transition to full enteral feeding, and prolonged hospital stay in gastroschisis has not been reported previously. In newborns with gastroschisis who underwent primary facial repair, thyroid functions should be controlled at appropriate times, and followed up closely.
\end{abstract}

Keywords: Gastroschisis, Enteral nutrition, Length of Stay, Congenital, Hypothyroidism, Newborn

\section{Introduction}

The term gastroschisis which is also named as abdominoschisis, laparoschisis, and paraomphalocele is derived from ancient Greek words for belly (gaster), and fissure (schisis). It is an anterior abdominal wall defect which is mostly localized on the right side with an incidence of 1 of 4000 births ve 4.4/10000 in live births (1-3). The defect is not covered by a sac and the rectus muscles meet in the midline at the xiphoid. This is an embryological developmental defect with male predominance (4). The etiology of gastroschisis is unknown but there is an association with young maternal age. There are two theories regarding the formation of gastroschisis. One theory suggests that involution of the right umbilical vein causes necrosis in the abdominal wall when physiological umbilical hernia is formed, leading to a right-sided defect. The second theory states that the right omphalomesenteric or vitelline artery prematurely involutes causing a weakening in the abdominal wall through which the intestinal contents protrudes out $(3,5)$. The organs are not enclosed in membranes; hence they are floating in the amniotic fluid resulting in perivisceritis, perivisceral adhesions, pseudomembranous covering, short mesentry, poor peristalsis and significant nutrient absorption imbalance (3).
Patients with gastroschisis can be divided into two groups as simple and complex gastroschisis (6). In complex gastroschisis, gastrointestinal anomalies such as intestinal atresia, stenosis, perforation, or volvulus may be seen. Simple gastroschisis is repaired by primary fascial closure and preformed silo while complex gastroschisis. In a recent UK study average hospitalization periods for simple, and complex gastroschisis have been reported as 24 days, and 47 days, respectively (6). Prolonged postoperative hospital stay in a patient with gastroschisis stem from complications as necrotizing enterocolitis, feeding difficulties and cholestatic jaundice, wound evisceration, wound infection, pneumonia and septicemia.

Congenital Hypothyroidism $(\mathrm{CH})$ is one of the most common preventable causes of mental retardation. Its worldwide incidence is 1:3000-4000 live births. Ideally universal screening at 3-4 days of age should be done so as to detect $\mathrm{CH}$. It accompanies mostly cardiac malformations, but comorbidities as neurologic abnormalities, cleft palate, and genitourinary malformation can be also seen. Increased incidence of hypothyroidism has been also reported among patients with Down syndrome.

Received: 29-01-2016, Accepted 15-02-2016, Available Online 15-03-2016

1 Dept. of Pediatric Surgery, Cengiz Gokcek Obstetrics and Children's Hospital, Gaziantep, Turkey

*Corresponding Author: Sevgi Buyukbese Sarsu E-mail: sarsusevgi@yahoo.com.tr 
Herein we report on $\mathrm{CH}$ as an unusual cause of delayed transition into full enteral feeding, and prolonged hospital stay in a newborn with gastroschisis

\section{Case}

A 15-year-old Syrian refugee mother $(\mathrm{G} 1, \mathrm{P} 1)$ gave birth to a male newborn with gastroschisis (weight,2100 g; p 90. ; length, $43 \mathrm{~cm}$, p 50, and head circumference, $31 \mathrm{~cm}, \mathrm{p} 75)$ via spontaneous vaginal delivery at $32 \mathrm{nd}$ gestational week in the Cengiz Gokcek Obstetrics and Children's Hospital who was then brought into male neonatal surgical care. His mother did not have any diseases during her pregnancy, and since she had not been followed up during antenatal period, she hadn't undergone ultrasonographic and serologic tests, and eventually her gastroschisis was not diagnosed till delivery.

Physical examination showed a large gastroschisis defect on the anterior abdominal wall to the right of an intact umbilical cord, without traces of membrane with an exteriorization of dilated bowel loops with swollen and thickened walls. Umbilical cord examination confirmed the presence of a single umbilical artery. All of the herniated contents were wrapped in moist and warm sterile dressings, umbilical venous catheterization was applied for fluid replacement, and antibiotic prophylaxis (ampicillin and gentamicin) was administered (Figure 1).

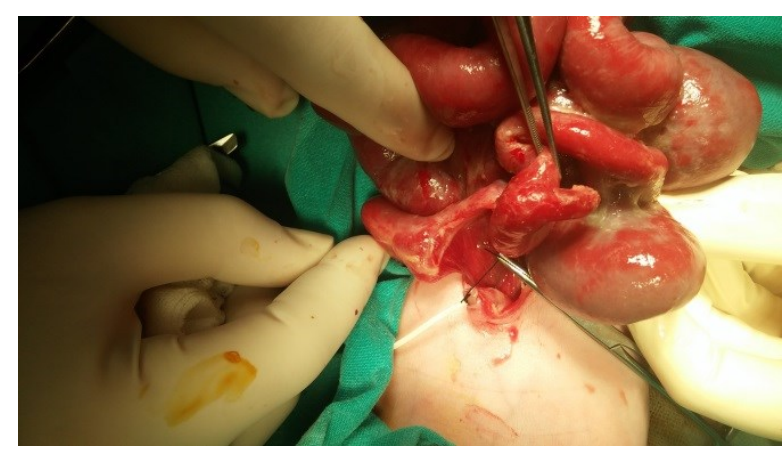

Figure 1. Umbilical vein catheterization for fluid replacement and antibiotic prophylaxis during preoperative period.

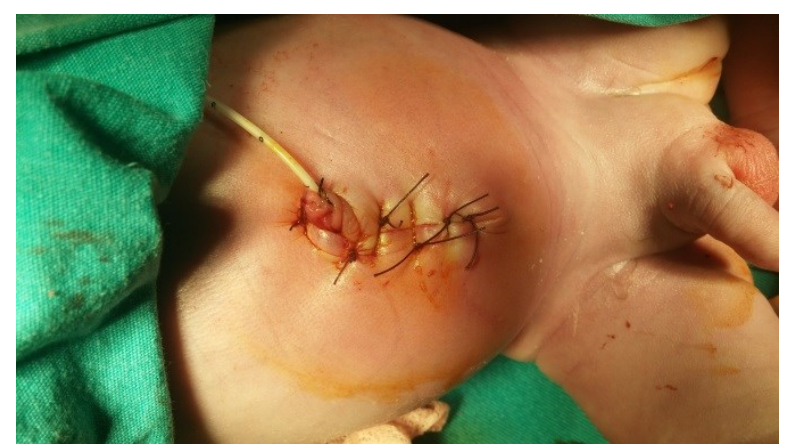

Figure 2. Primary repair of gastroschisis.

Medical Science and Discovery 2016; 3(3): 137-40
Surgical procedures were performed under general anesthesia after tracheal intubation, and prevention of hypothermia. Incision was extended vertically on either side for $2 \mathrm{~cm}$. Intestinal tract was not atretic. There were no other abnormalities in the newborn. Abdominal wall defect was primarily closed without the need for silo reduction (Figure 2). Although on routine screening test TSH value was detected as 70 $\mu \mathrm{IU} / \mathrm{ml}$, since the test was performed within the first 24 hours of life this result was deemed to be false positive

The patient was kept on mechanical ventilation for 2 days. Total parenteral nutrition started. Enteral nutrition was started at 6 days of life but could not be progressively increased in amount. He was receiving orally only breast milk, and after three feedings he vomited once (non-bilious vomitus) after every three feeds, and defecated scarce amount stool once a day. Besides, abdominal distension was detected on 15 th day of his life, she was consulted to pediatric endocrinology, and levothyroxine therapy was initiated when higher levels of free T3 [2.10 (2.3-5.0) $\mathrm{pg} / \mathrm{ml}]$, free T4 $[0.67(0.80-2.0) \mathrm{ng} / \mathrm{dl}]$, and TSH [150 (0.72-11) $\mu \mathrm{IU} / \mathrm{ml}]$ were detected. Thyroid US was unremarkable. After treatment, the patient could tolerate full enteral feeding, so he was discharged on postoperative 20th day without complication

Written informed consent was obtained from the patient's legal guardian(s) for the publication of this case report. The study was approved by the local ethics committee (Conclusion no:19.11.2015/27).

\section{Discussion}

Gastroschisis is a neonatal disease with a gradually increasing incidence (7). Although its pathogenesis is not known precisely yet, gynecological immaturity and poor nutritional status have been associated with this neonatal congenital (8). Our case was a 15 -yearold Syrian refugee. Delay in the transition to full enteral feeding because of intestinal dysfunction in newborns with gastroschisis is a major neonatal morbidity. It results in prolonged hospital stay, and longer administration of TPN, development of sepsis, and infection. In previous studies, shorter duration of TPN, and hospital stay, lower rates of infectious complications, and more favorable prognosis have been reported in newborns whose enteral feeding was initiated at an early stage. (9).

Enteral feeds are usually initiated at a low volume and its volume is gradually increased if tolerated. Our case gradually started to receive breast milk. Progressive increase in the frequency, and amount of breast feeding could not be achieved, Due to newborn's intolerance to milk Clinical criteria for initiation of enteral feeding in cases with gastroschisis include definitive closure of the defect, detection of nonbilious nasogastric drainage fluid, maintenance of regular 
defecation pattern, and absence of abdominal distension (9). In consideration of these criteria, enteral feeding was initiated on 6. day of newborn's life. In newborns who started to receive enteral feeds within the first 7 days of their lives length of hospitalization, and days on TPN decreased, and infectious complications as fever were less frequently encountered when compared with patients whose enteral feeds were initiated 21 days after closure of the abdominal defect (9). Bucher et. al. found that time to closure was associated with earlier age of full enteric feeding and decreased TPN duration (10). Cases that underwent primary closure start to receive enteral feeding earlier than those treated with silo reduction procedure. In a 2013 study, Baud et al. showed that outcomes were improved for those who started to receive oral enteral nutrition before 37 weeks, and incidence of intestinal atresia, necrosis or perforation also decreased (11). Prolonged exposure to amniotic fluid may damage intestinal wall. Our case with gastroschisis was delivered spontaneously at 32 . gestational week via vaginal route. However in another study, any difference in transition to enteral feeding was not detected between newborns delivered before or after 37 . gestational week. (12).

A newborn with gastroschisis should be immediately referred to surgery for the repair of abdominal wall defect. Primary fascial closure of the abdominal wall defect has outcomes superior to silo reduction procedure, and it should be considered in all newborns with gastroschisis without increased intraabdominal pressure, and suitable abdominal anatomy (13). Our case was suitable for primary fascial closure. Studies of gastroschisis typically focus on the surgical complications which may develop in a newborn with gastroschisis include necrotizing enterocolitis, feeding difficulties, and cholestatic jaundice, wound evisceration, and infection, pneumonia, and septicemia ( $14-16$ ). Besides, in $26.7 \%$ of the cases with gastroschisis developmental delay was detected based on BSID III criteria (17). In the same study it was reported that all cases with developmental delay, and transient hypothyroidism had received povidone iodine (PVP-I) dressings, and undergone silo operations before Transient hypothyroidism may be caused by maternal or neonatal factors. Maternal factors include anti-thyroid medications, trans placental thyrotropin receptor blocking antibodies and iodine deficiency or excess. Our case had not maternal factors. Neonatal factors include, neonatal iodine deficiency or excess, congenital liver hemangiomas and mutations in the genes encoding for DUOX and DUOXA2. We didn't perform genetic tests in our case. Transient neonatal hypothyroidism may develop secondary to giant omphalocele which is a prevalent anterior wall defect, escharification of omphalocele sac, long-term use of PVP-I for escharification, and epithelialization of omphalocele, and hyper- permeability of neonatal tissues. Topical PVP-I can rarely induce this condition. In this case if omphalocele sac atrofies, then iodine exerts minimal systemic effects, and since transient thyroid dysfunction develops, thyroid supplementation is not required Delay in transition to full enteral feeding, and prolonged hospital stay in association with $\mathrm{CH}$ have not been reported in cases with gastroschisis before.

Signs and symptoms of hypothyroidism include postmaturity, macrosomia or wide open posterior fontanel at birth or abdominal distension, constipation, poor feeding, prolonged jaundice, hypotonia, hoarse cry, umbilical hernia, macroglossia, or dry edematous skin in infancy. In our case the first three clinical symptoms were detected. In the absence of newborn screening programs, the diagnosis of $\mathrm{CH}$ is made after development of clinical manifestations. Thyroid function tests should be performed on all newborns. In our hospital routine screening tests are being performed.. The time at which the sample is taken may vary between centers, with the majority taking blood from a heel prick at 24 hours of age to minimize the false-positive higher TSH measurement due to the physiological neonatal TSH surge that elevates TSH levels and causes dynamic T4 and T3 changes in the first 1 or 2 days after birth. Early discharge of mothers after delivery has increased the ratio of false- positive TSH elevations. If this is not possible, testing should be performed before discharge or within seven days of birth. False-positive TSH elevations may be found in specimens collected at 24 to 48 hours after birth, and false-negative results may be also seen. However in this case blood samples of this newborn were drawn to screen TSH, and T4 levels within the first 24 hours of his life, and increased TSH level was thought to be a false-positive result, and so overlooked. A minority of patients develop $\mathrm{CH}$ as a result of a hereditary defect in thyroid hormone biosynthesis. As was the case with our patient, this condition progresses with low T4 and elevated TSH levels. In this case levothyroxine (Lthyroxine) should be initiated at daily doses of 10-15 $\mu \mathrm{g} / \mathrm{kg}$ as soon as the diagnosis was made. The 1thyroxine tablet should be crushed, mixed with breast milk, formula or water and fed to the infant. Regular monitoring should be performed.

\section{Conclusion}

Delay in the transition to full enteral feeding, and prolonged hospital stay in a newborn who underwent primary repair for gastroschisis may be due to $\mathrm{CH}$. Even if silo operation is not performed, screening tests for $\mathrm{CH}$ should be performed between $2 \mathrm{th}$, and 4th Post-natal days, and the patient should be closely followed-up.

Acknowledgments: This research received no specific grant from any funding agency, commercial or not-for-profit sectors. 
Conflict of Interest: The authors declare no potential conflicts of interest with respect to the research, authorship, and/or publication of this article.

\section{References}

1. Dharmraj $M$ and Verma AP.Gastroschisis associated with lower limb and spinal congenital anomalies. J clin Neonatol. 2012; 1(4);217- 20.

2. Kirby RS, Marshall J, Tanner JP, Salemi JL, Feldkamp ML, Marengo L, et al. The National Birth Defects prevention Network, Prevalence and correlates of gastroschisis in 15 U.S. states: 1995- 2005. Obstet Gynecol. 2013;122: 275-81.

3. Ionescu S, Mocanu M, Andrei B, Bunea B, Carstoveanu C,Gurita A, Tabacaru R, Licsandru E,S tanescu D, Selleh M. Differential diagnosis of abdominal wall defectsomphalocele versus Gastroschisis. Chirurgia. 2014;109(1):7-14.

4. Afsarlar CE, Kendirci HNP, Erdogan D, Ozguner IF, Cavususoglu YH,Karaman A,Cetinkaya S. The first case of bruck syndrome associated with gastroschisis. The Turkish Journal ofPediatrics 2013;55:651-4.

5. Emily R. Christison-Lagay a, Cassandra M. Kelleher b, Jacob C. Langer a, Seminars in Fetal \& Neonatal Medicine 16. $2011 ; 164-72$.

6. Bradnoch TJ,Marven S, Owen A, Johnson P, Kurinczuck JJ, Spark P, Draper ES, Knight M. Gastroschisis: one year outcomes from national cohort study.BMJ.2011;343:67 49.

7. Overcash R, DeUgarte D, Stephenson M, Gutkin R, Norton M, Parmar S, et al. Factors associated with gastroschisis outcomes. Obstet Gynecol. 2014; 124: 551-7.

8. Gill SK, Broussard C, Devine O, Green RF, Rasmussen S a, Reefhuis J. Association between maternal age and birth defects of unknown etiology_-United States, 1997-2007. Birth Defects Res A Clin Mol Teratol 2012;000.
Aljahdali A, Mohajerani N, Skarsgard E. Effect of timing of enteral feeding on outcome in gastroschisis. J Pediatr Surg. 2013; 48: 971-6.

10. Bucher B, Mazotas I, Warner B, Saito J. Effect of time to surgical evaluation on outcomes of infants with gastroschisis. J Pediatr Surg. 2012; 47: 1105-10.

11. Baud D, Lausman A, Alfaraj M, Seaward G, Kingdom J, Windrim R, et al. Expectant management compared with elective delivery at 37 weeks for gastroschisis. Obstet Gynecol. 2013; 121: 990-8.

12. Harris J, Poirier J, Selip D, Pillai S, Shah AN, Jackson CC, Chiu B. Early Closure of Gastroschisis After Silo Placement Correlates with Earlier Enteral Feeding. Journal of Neonatal Surgery 2015; 4(3):28.

13. Chesley PM, Ledbetter DJ, Meehan JJ, Oron AP, Javid PJ. Contemporary trends in the use of primary repair for gastroschisis in surgical infants. Am $J$ Surg. 2015;209(5):901-5.

14. Wood S, Samangaya R, Gillham J, Morabito A. Gastroschisis and the risk of short bowel syndrome: outcomes and counselling. Neonatol. 2014; 105: 5-8.

15. Charlesworth P, Akinnola I, Hammerton C, Praveena P, Desai A, Patel S, et al. Preformed silos versus traditional abdominal wall closure in gastroschisis: 163 infants at a single institution. Eur J Pediatr Surg. 2014; 24: 88-93.

16. Alsheri A, Emil S, Laberge J, Skargard E. Outcomes of early versus late intestinal operations in patients with gastroschisis and intestinal atresia: results from a prospective national database. J Pediatr Surg. 2013; 48: 2022-6.

17. Sirichaipornsak S , Jirapradittha J, Kiatchoosakun P, Suphakunpinyo C. Neurodevelopmental outcomes of children with gastroschisis at university hospital in northeast Thailand. Asian Biomedicine. 2011; 5:6: 861-6. 